the least expense of stimulation. The diuretic essential oils and scoparium I object to as being too stimulating. Digitalis is dangerous in two ways; first, because there is a risk of setting up uterine contractions; and, secondly, because digitalis will sometimes kill the foetus in utero by producing paralysis of the heart. I have seen this occur. We are not concerned here with the other indication of treatment. The form of diuretic I would use would be the acetate of potassa largely diluted, combined with the vinegar of squill, and full doses of tincture of henbane. It was taught by the late Dr. Golding Bird that acetate of potassa was the best diuretic salt in the pharmacopœia; and he especially insisted on the practice of dilution. The urine appears to be more readily made alkaline by this salt than by the other salts of potassa; the nitrate passes out of the body as nitrate; liquor potassæ is less diuretic and is apt to throw down the phosphates of the urine while the bladder is still full; and the same is true of the carbonate; the bicarbonate may be used if the stomach rejects the acetate It should be remembered that it is not diuresis only that is required, but a copious secretion of bland urine, at the expense of as little stimulation to the kidney as possible.

Hyperæesthesia of the gastric nerves is a very embarassing complication of pregnaney. The danger to both mother and foetus is very considerable; indeed, it often happens that one must fall a sacrifice to the other. The vomiting which declares this irritable state of the nerves of the stomach is only, so far as I am aware, capable of being dealt with by two methods of treatment as long as the foetus remains in utero. The first is the resolute use of morphia and hydrocyanic acid; and the second is a dietetic system, of which I will speak presently. A radical error is generally committed in the administration of opiates in the gastric irritability of pregnancy, and in other cases also where it is desired to allay vomiting. The bulk of the dose is almost invariably too great, the sedative is given too much diluted, and the quantity of fluid is resented by the stomach. It should be borne in mind that a medicine used for the purpose in question is essentially a lotion to the coats of the stomach. If the lotion is made too weak the intent is frustrated. The bulk of the dose should never exceed one fluid drachm, and it may be made less with advantage. Half a grain of acetate of morphia, three drops of the dilute hydrocyanic acid, and one of Fleming's tincture of aconite, to a drachm or even half a drachm of water, will not only burthen the stomach less, but will produce a greater topical effect on the nerves of the stomach than if given in the usual state of dilution. The food should be given in one of two ways; if taken in any considerable quantity, i.e., if any attempt at a meal be made, it should be eaten soon after the exhibition of a dose of the medicine, and should be soon followed by another; or the diet should consist of single spoonfuls of milk, strong beef tea, or egg-brandy, often repeated; or very frequent doses of protein. The most remarkable case of vomiting I ever heard of was successfully treated by five grain doses of protein given in a teaspoonful of milk every hour; the case occurred some years ago, and was recorded in the journals of the time, but my memory will not serve me for a reference. The dietetic principle seems to be either to overpower the hyperæsthesia immediately before eating; or to give food in small quantities, and in such a form as to pass readily into the duodenum, thus diminishing the offence to the stomach, and throwing the task of digestion mainly on the small intestines. It is of course only possible within these limits to do more than indicate the general plan of treatment or the principles upon which to act. Modifications easily arise when the main principle is grasped.

The last eccentric irritation to which I shall refer is hyperæsthesia of the mammæ. Mere irritability of one or both breasts may obtain, or the altered innervation of the part may be succeeded by great vascularity and induration; and in some extreme cases suppuration takes place. There can be little doubt but that these affections of the breast, which in the first instance derive from the irritation of the ovum contained in the uterus, are again reflected upon that organ. A brisk saline aperient and leeching, followed by the application of a belladonna plaster, will be found the best method of treatment.

The preceding remarks are a mere sketch of eccentric medication. In the next paper will be discussed centric medication the properties of the agents used, and the circumstances which call for, or disallow, their exhibition.

[To be continued.]

\section{CASES OF POISONING BY WHITE PRECIPITATE, AND BY POPPY-HEAD.}

By W. H. Michael, Esq., Swansea.

[Read before the Monmouthshire and South Wales Branch, July 23rd,1857.]

I. POISONING BY WhITE PRECIPITATE.

Miss P., a schoolmistress, aged 37 years, on December 27th, 1856 , bought, at three druggists' shops, fourpennyworth of white precipitate, for a suicidal purpose. She obtained, as it would appear from the statement of the tradesmen who supplied her, 140 grains of ammonio-chloride of mercury, two samples of which, when analysed at the shops, were found to be absolutely pure. At or about 8 o'clock in the evening of the same day, she ate a large portion of the powder spread on bread and butter. About ten grains, adulterated with sulphate of lime, were found on the plate which she had used, and on some portion of the bread and butter she had been unable to eat, and had thrown away. Judging from her own statements upon several occasions, and from the foregoing facts, it appears probable that not less than one hundred grains were swallowed. She was seen by me about three hours and a half afterwards, and was then lying on the floor of a bedroom, complaining of great pain in the region of the stomach, and severe intermitting cramp of the left side and lower extremities, which came on at intervals of two or three minutes. The pulse was very rapid, weak, and thready; the surface cold, and covered with a clammy perspiration; the countenance was pale, drawn, and anxious; the tongue very red; the pupils were dilated, and contracted but slowly under the stimulus of light. She had vomited very freely a thick tenacious mucus, of a white colour, with white sediment. This I was unable to examine, as it had been thrown away previously to my arrival. About half an hour afterwards, the bowels were very freely acted on, an enormous quantity of foetid frecs passing, sufficient to fill two chamber utensils. Several pieces of white precipitate were picked off the tongue, which had apparently been left there by the action of vomiting, which was kept up by diluent drinks for two hours. Great prostration continued for several hours, and the pain in the stomach continued for three or four days, when it gradually subsided. On Dec. 28th, reactionary fever set in, which, however, was never severe, the pulse seldom ranging beyond 120 , and the head remaining unaffected: indeed, it appeared as though the thorough unloading of the alimentary canal had been productive of great benefit to the general health; the appearance of Miss P. after her recovery, which took place in a fortnight, being better than it had been for some years. No salivation occurred. The treatment adopted consisted of emetics, diluents, and sedatives, with counterirritation and application of external heat until reaction set in, when ordinary salines, etc., followed by quinine, were employed.

The features of interest in the case are, the large quantity taken, and the comparative immunity from alarming symp toms, which may probably have been owing to the vehicle (butter) shielding the coats of the stomach, and disarming the poison of some of its noxious effects.

\section{FATAL POISONING BY A POPPY-HEAD.}

On February 27 th last, two children, twins, about three months old, were brought to me about 11 A.M., evidently suffering from narcotism. It appeared, upon inquiry, that the mother had the night before, with the view of procuring sleep, boiled a poppy-head in a small saucepan, and given each child one or two teaspoonfuls of the decoction. Stimulants, coffee, etc., were administered; but one child died the same evening, seven or eight hours after it was first seen. The poppyhead was shown me, and a few drops of the remaining decoction, which had the characterisic opium smell. No tests were applied.

\section{CASE OF CONCEALED ACCIDENTAL UTERINE HÆAMORRHAGE.}

By John Audland, Esq., Tintern, near Chepstow.

ON Sunday afternoon, August 30th, I was sent for to visit Mrs. M., aged 42 , who expected her tenth confinement in three or four weeks. I learned on that and subsequent visits, that on the previous Wednesday night, soon after getting into bed, she became very faint and cold, and had a severe fit of shivering. Some gin was given her, and she gradually rallied. The next morning she got up, but felt very weak and giddy, 